ARTICLE

Received 10 Mar 2016 | Accepted 7 Jul 2016 | Published 12 Sep $2016 \quad$ DOl: 10.1038/ncomms12525

\title{
The Arabidopsis MIEL1 E3 ligase negatively regulates $A B A$ signalling by promoting protein turnover of MYB96
}

Hong Gil Lee ${ }^{1} \&$ Pil Joon Seo ${ }^{1}$

The phytohormone abscisic acid ( $A B A$ ) regulates plant responses to various environmental challenges. Controlled protein turnover is an important component of $A B A$ signalling. Here we show that the RING-type E3 ligase MYB30-INTERACTING E3 LIGASE 1 (MIEL1) regulates ABA sensitivity by promoting MYB96 turnover in Arabidopsis. Germination of MIEL1-deficient mutant seeds is hypersensitive to $A B A$, whereas MIEL1-overexpressing transgenic seeds are less sensitive. MIEL1 can interact with MYB96, a regulator of ABA signalling, and stimulate its ubiquitination and degradation. Genetic analysis shows that MYB96 is epistatic to MIEL1 in the control of ABA sensitivity in seeds. While MIEL1 acts primarily via MYB96 in seed germination, MIEL1 regulates protein turnover of both MYB96 and MYB30 in vegetative tissues. We find that $A B A$ regulates the expression of MYB30-responsive genes during pathogen infection and this regulation is partly dependent on MIEL1. These results suggest that MIEL1 may facilitate crosstalk between ABA and biotic stress signalling.

\footnotetext{
${ }^{1}$ Department of Biological Sciences, Sungkyunkwan University, Suwon 16419, Republic of Korea. Correspondence and requests for materials should be addressed to P.J.S. (email: pjseo1@gmail.com).
} 
A s sessile organisms, plants have evolved sophisticated biochemical and physiological responses to deal with environmental challenges, such as drought, high salinity, wounding and temperature extremes ${ }^{1}$. The phytohormone $\mathrm{ABA}$ contributes in part to plant adaptation to environmental fluctuations by modulating a wide array of physiological processes, including seed dormancy and germination, early seedling growth, guard cell functioning and stress tolerance ${ }^{2-5}$.

$\mathrm{ABA}$ is perceived by the receptor proteins PYRABACTIN RESISTANCE 1 (PYR)/PYR1-LIKE (PYL)/REGULATORY COMPONENTS OF ABA RECEPTOR ${ }^{6,7}$. In the presence of $\mathrm{ABA}$, these receptors interact with protein phosphatase $2 \mathrm{C}$ (PP2C) proteins such as ABA-INSENSITIVE 1 (ABI1) and ABI2, which negatively regulate $\mathrm{ABA}$ signalling, and inhibit their catalytic activities ${ }^{8}$. Subsequently, SNF1-RELATED KINASE $2 \mathrm{~s}$ are derepressed from $\mathrm{PP} 2 \mathrm{Cs}$ and phosphorylate leucine-zipper ABA-responsive element (ABRE)-binding proteins/ABREbinding factors (AREBs/ABFs), which bind directly to ABREs of stress-responsive genes, to stimulate their transcriptional activities $^{8,9}$.

In addition to AREBs/ABFs, MYB and MYC transcription factors also constitute ABA-dependent signalling pathways. MYB2 and MYC2 cooperatively regulate the drought-responsive RESPONSIVE TO DESSICATION 22 (RD22) gene by directly binding to its promoter ${ }^{10,11}$. Several R2R3-type MYB transcription factors, including MYB15, MYB20, MYB41, MYB44 and MYB96, also mediate ABA signalling and regulate a variety of physiological responses under stress conditions, such as root and shoot development, hormone metabolism, cell expansion, drought tolerance and cuticular wax biosynthesis ${ }^{12-18}$, underscoring the importance of transcriptional regulation in plant stress responses.

Transcription factor activities are further shaped by additional post-translational modifications. In particular, the ubiquitination process is a representative way of modulating protein turnover and is mediated by the sequential action of three enzymes: Ub-activating enzyme (E1), Ub-conjugating enzyme (E2) and Ub ligase (E3) ${ }^{19-21}$. Notably, the Arabidopsis genome encodes $>1,500$ E3 enzymes ${ }^{19,20}$, and this large number of E3 ligases implies specific recognition of target substrates ${ }^{22}$.

Among the Arabidopsis E3 ligases, $>470$ proteins belong to the RING-finger protein family ${ }^{20,23}$. Remarkably, a significant number of the members are responsible for $A B A$ signal transduction $^{22,24,25}$. For instance, the RING-type E3 ligase RING FINGER OF SEED LONGEVITY 1 stimulates protein turnover of PYR4 and PYR1 at the plasma membrane ${ }^{26}$. RING-type E3 ligase ABI3 INTERACTION PROTEIN 2 (AIP2) interacts with and targets ABI3 for protein degradation ${ }^{27}$. ABI5 is also proteolytically degraded by RING-finger protein KEEP ON GOING, ensuring a low level of ABI5 production in the absence of $\mathrm{ABA}^{28,29}$. Protein stability of ABA-inducible DEHYDRATION-RESPONSIVE ELEMENT BINDING PROTEIN2A (DREB2A) is also controlled by C3HC4 RING-type E3 ligases DREB2A-INTERACTING PROTEIN 1 (DRIP1) and DRIP2 (ref. 30). In addition, the SALT- AND DROUGHT-INDUCED RING FINGER 1 is a positive regulator of $\mathrm{ABA}$ signalling in seed germination, stomatal closure and drought tolerance ${ }^{31}$. The RING-H2 E3 ligases RHA2a and RHA2b also regulate ABA-dependent seed germination, early seedling development and drought tolerance through an ABI3/4/5-independent pathway ${ }^{32,33}$.

In this study, we demonstrate that the RING-type E3 ligase MIEL1, which was previously reported as a negative regulator of hypersensitive cell death ${ }^{34}$, is involved in ABA regulation of seed germination by promoting MYB96 turnover in Arabidopsis. We found that MIEL1-deficient mutant seeds are hypersensitive to $\mathrm{ABA}$, whereas MIEL1-overexpressing transgenic seeds (35S:MIEL1-MYC) exhibit a hyposensitive response during the germination. The MIEL1 function in ABA-modulated seed germination depends primarily on MYB96. However, in vegetative stages, MIEL1 triggers simultaneous protein turnover of MYB96 and MYB30, and we show that ABA can influence expression of MYB30-regulated genes related to biotic stress. We propose that MIEL1 coordinates ABA-dependent adaptive responses and defensive hypersensitive responses in Arabidopsis.

\section{Results}

MIEL1 regulates ABA-dependent seed germination. Ubiquitination is a representative molecular mechanism that regulates ABA signalling $20,22,24$. To further understand the ABA signalling network, we analysed the ABA responsiveness of several E3 ligase mutant seeds to identify additional regulator(s) of the process ${ }^{35}$.

Among the mutants examined, we preliminarily found that miel1-1 seeds were hypersensitive to ABA during germination (Fig. 1a). To validate the results of our screen, we obtained an additional mutant allele miel1-2 and also generated MIEL1-overexpressing 35S:MIEL1-MYC transgenic plants (Supplementary Fig. 1). The germination kinetics of the miel1 mutant and 35S:MIEL1-MYC transgenic seeds were comparable to that of wild-type seeds in the absence of ABA (Fig. 1b; Supplementary Fig. 2). However, in the presence of ABA, germination of miel1-1 and miel1-2 mutant seeds was significantly delayed, whereas the 35S:MIEL1-MYC transgenic seeds exhibited reduced ABA sensitivity (Fig. 1b; Supplementary Fig. 2). The differences in the germination timing of the MIEL1-deficient mutant and 35S:MIEL1-MYC transgenic seeds were maintained at higher ABA concentrations (Fig. 1b; Supplementary Fig. 2). Although the impact of MIEL1 in ABA-dependent seed germination was not strong as much as that of ABIl, its role in seed germination was still relevant and significant (Supplementary Fig. 2).

To support the roles of MIEL1 in seed germination, we analysed the transcript accumulation of MIEL1 in seeds. Expression of MIEL1 in dry seeds was comparable to that in 7-day-old seedlings (Supplementary Fig. 3). In addition, transcript accumulation of MIEL1 was substantially elevated upon the imbibition and stratification (Supplementary Fig. 3). Furthermore, MIEL1 was induced by gibberellic acid (GA) and the ABA synthesis inhibitor fluridone, which accelerate seed germination, whereas $\mathrm{ABA}$ and the GA synthesis inhibitor paclobutrazol suppressed MIEL1 in seeds (Fig. 1c). These observations are consistent with public expression data (eFP browser $)^{36}$, which shows that the MIEL1 gene is strongly expressed in micropylar endosperm (Supplementary Fig. 4). The micropylar endosperm surrounding the radicle tip acts as a physical barrier of seed germination and is the major place, where antagonistic actions of ABA and GA (or ethylene) are supposed to regulate endosperm weakening and thus endosperm rupture/ radicle protrusion ${ }^{36-38}$. Given that the $26 \mathrm{~S}$ proteasome activity is required for micropylar endosperm autolysis ${ }^{39}$, MIEL1 might play a role in this process.

MIEL1 can interact with MYB96. MIEL1 is an E3 Ub ligase that commonly facilitates protein degradation through physical interactions with a target substrate ${ }^{34}$. Therefore, we asked which protein might be a regulatory target of MIEL1 in the regulation of ABA sensitivity. We performed a yeast-two-hybrid (Y2H) screen and identified a physical interaction with a partial fragment of MYB96, a key mediator of ABA signalling in a variety of physiological processes ${ }^{15-17,40-42}$. To validate this observation, the full-size MYB96 gene was fused in-frame to the $3^{\prime}$-end of 
a
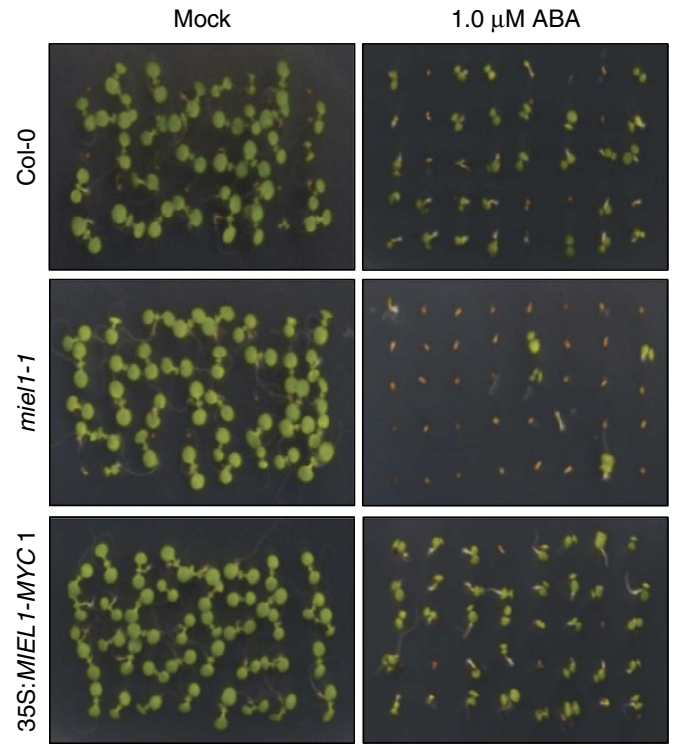

C

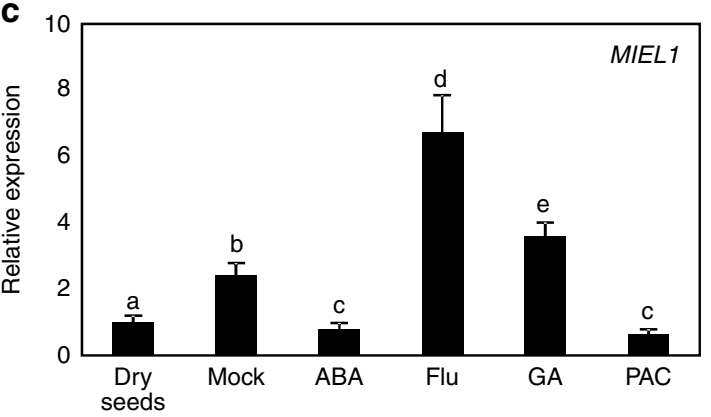

b
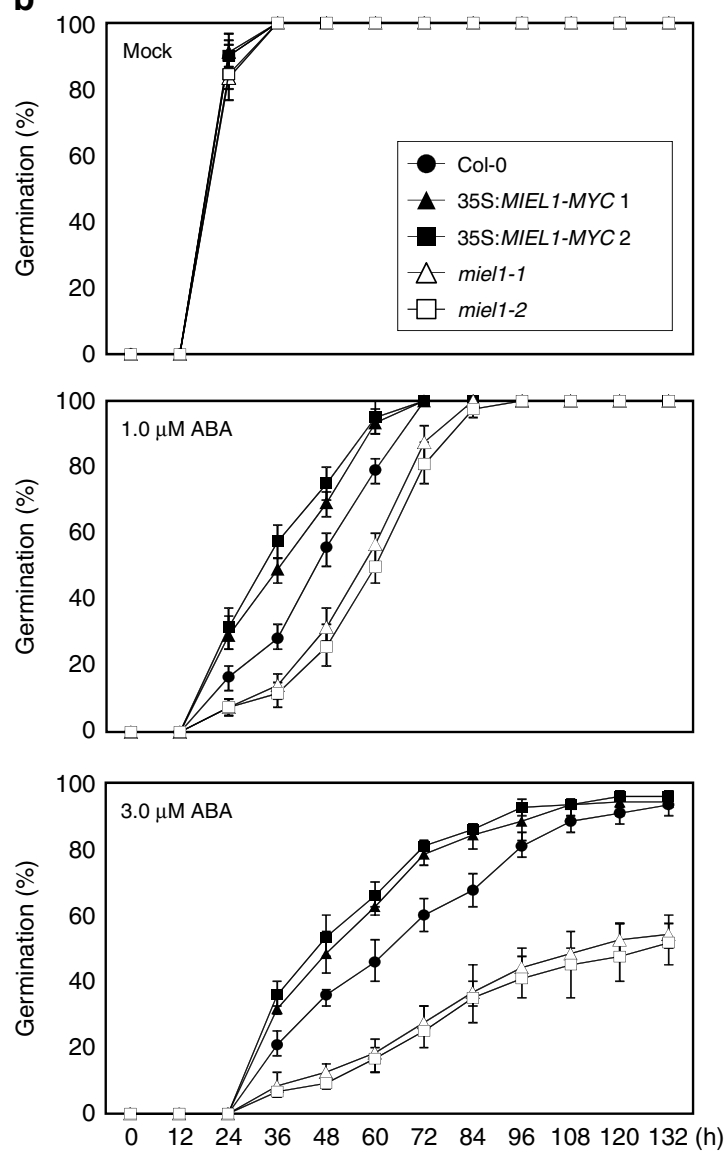

Figure 1 | ABA sensitivity of 35S:MIEL1-MYC transgenic and miel1-deficient seedlings during germination. (a) Altered ABA sensitivity of 35S:MIELT-MYC and miel1-1 early seedlings. Seeds were germinated and grown on $1 \mu$ M ABA under long-day (LD) conditions. Photographs were taken 3 days after cold stratification. (b) Germination percentage. The percentage of seed germination of the indicated genotypes grown on different concentrations of ABA was quantified after the end of stratification. Radicle emergence was used as a morphological marker for germination. At least 50 seeds per genotype were measured in each replicate. Biological triplicates were averaged. Bars indicate the s.e.m. (c) MIEL1 expression in seeds. Seeds were germinated on MS medium supplemented with $1 \mu \mathrm{M} \mathrm{ABA}, 10 \mu \mathrm{M}$ fluridone (Flu), $0.5 \mu \mathrm{M}$ GA or $15 \mu \mathrm{M}$ paclobutrazol (PAC) and incubated for 2 days under LD conditions. Transcript accumulation was analysed by RT-qPCR. Three independent biological replicates were averaged. Different letters represent a significant difference at $P<0.05$ (one-way analysis of variance with Fisher's post hoc test). Bars indicate the s.e.m.

activation domain (AD) of GAL4, and the construct was co-expressed with GAL4 DNA-binding domain (BD)-MIEL1 fusion construct in yeast cells. As expected, MYB96 interacted with MIEL1 in yeast cells (Fig. 2a).

To identify the regions responsible for the interaction between MIEL1 and MYB96, we constructed a series of deletion forms of these two proteins (Fig. 2b). Y2H analysis revealed that the $\mathrm{N}$-terminal region of MYB96 (residues 1-143) was responsible for the interaction between MIEL1 and MYB96. Likewise, MYB96 specifically interacted with the N-terminal region of MIEL1 (residues 1-140; Fig. 2c).

To further support the interaction of MIEL1 with MYB96 in vivo, we also performed bimolecular fluorescent complementation (BiFC) analysis using Arabidopsis protoplasts. The MYB96 complementary DNA (cDNA) sequence was fused in-frame to the $5^{\prime}$-end of a gene sequence encoding the C-terminal half of YFP, and the MIEL1 gene was fused in-frame to the $5^{\prime}$-end of a sequence encoding the N-terminal half of YFP. The fusion constructs were then transiently co-expressed in Arabidopsis protoplasts. Yellow fluorescence was exclusively detected in the nucleus (Fig. 2d), while co-expression with an empty vector did not show any discernible fluorescence (Fig. 2d; Supplementary Fig. 5). The in planta interaction of MIEL1 and MYB96 was also verified by co-immunoprecipitation (Co-IP) assay using Nicotiana benthamiana cells transiently co-expressing MIEL1MYC and MYB96-GFP fusion constructs (Fig. 2e).

We then asked whether the MIEL1-MYB96 interactions are mutually specific in the context of ABA signalling components. Several E3 Ub ligases responsible for ABA signal transduction, including AIP2, ATL43, ARM REPEAT PROTEIN INTERACTING WITH ABF2, CARBOXYL TERMINUS OF HSC70-INTERACTING PROTEIN and XERICO ${ }^{43}$, were employed to generate GAL4 BD fusion constructs, and the fusion constructs were used to coexpress with the GAL4 AD-MYB96 fusion construct in yeast cells. No discernible interaction was observed among the diverse combinations (Supplementary Fig. 6). Furthermore, MIEL1 was primarily associated with MYB96, and other transcriptional regulators were not preferred as interactive targets (Supplementary Fig. 6), suggesting a specific interaction between MIEL1 and MYB96 among components of ABA signalling networks.

MIEL1 ubiquitinates MYB96 and facilitates its degradation. The physical interactions of MIEL1 with MYB96 led us to examine whether MIEL1 ubiquitinates MYB96. To examine 
a

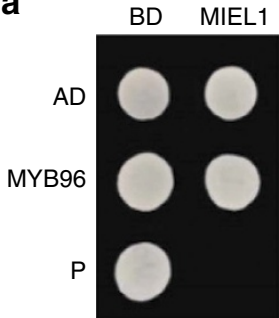

-LW

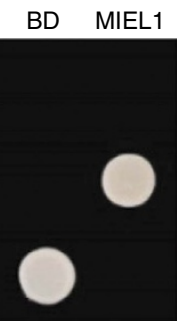

-LWHA b

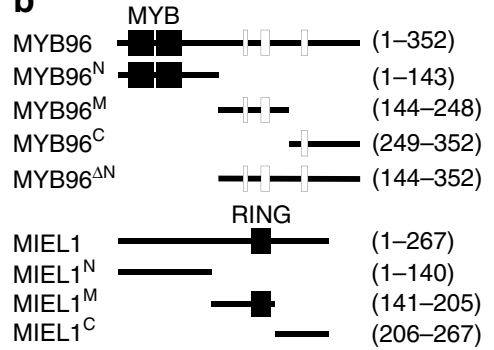

d
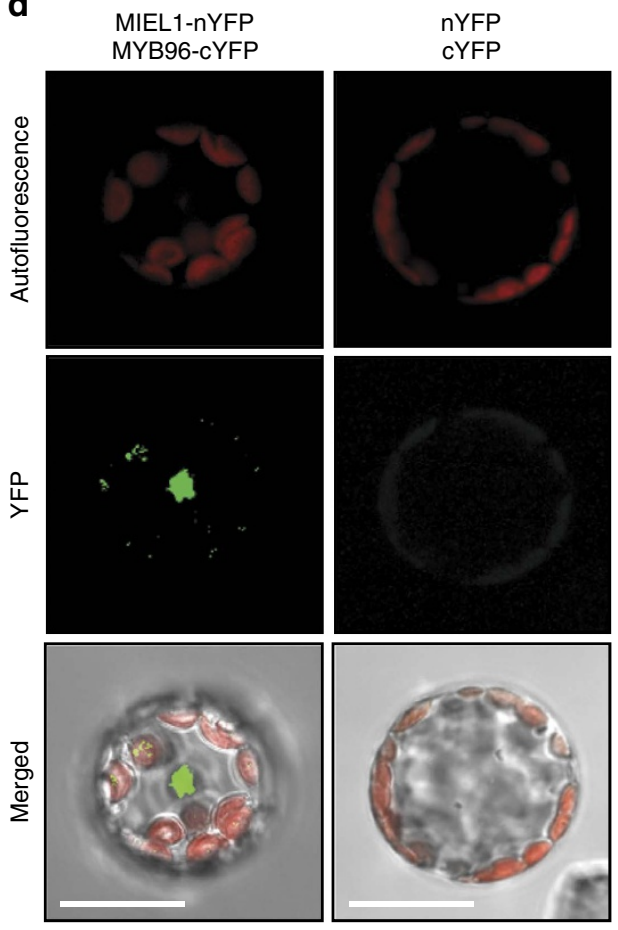

e

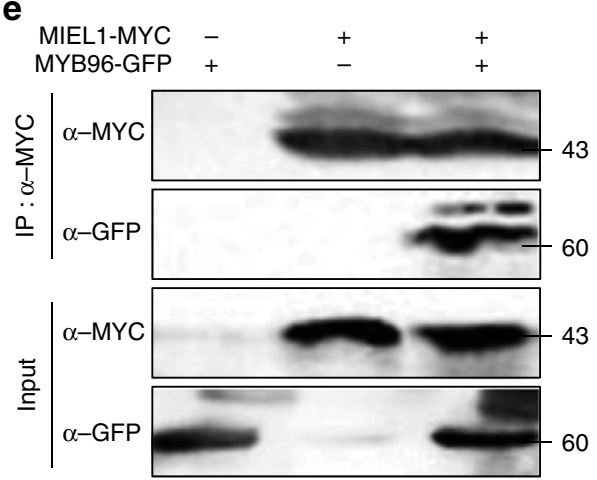

Figure 2 | Interaction of MIEL1 with MYB96. (a) Yeast-two-hybrid (Y2H) assays. Y2H assays were performed with the MIEL1 protein fused with the DNA-binding domain (BD) of GAL4 and MYB96 fused with the transcriptional activation domain (AD) of GAL4 for the analysis of their interactions. Interactions were examined by the cell growth on selective media. -LWHA indicates Leu, Trp, His and Ade drop-out plates. -LW indicates Leu and Trp drop-out plates. GAL4 was used as a positive control (P). (b) Deletion constructs of MIEL1 and MYB96. Numbers indicate residue positions. (c) Interaction domain mapping. $\beta$-Galactosidase ( $\beta$-Gal) activity was quantified after growing yeast strains in liquid culture with 0 -nitrophenyl- $\beta$ - $D$-galactopyranoside as a substrate. Three independent measurements of $\beta$-Gal activities were averaged and statistically analysed by a Student's $t$-test ( $\left.{ }^{\star} P<0.05\right)$. Bars indicate the s.e.m. (d) BiFC assays. Partial YFP fusion constructs containing either MIEL1 or MYB96 were transiently co-expressed in Arabidopsis protoplasts. Chloroplasts appear in red. Scale bars, $20 \mu \mathrm{m}$. (e) Co-immunoprecipitation assays. A. tumefaciens cells containing MIEL1-MYC and MYB96-GFP constructs were injected to 3-week-old N. benthamiana leaves. Epitope-tagged proteins were detected immunologically using corresponding antibodies. The molecular weight $(\mathrm{kDa})$ was indicated on the right side of the gel. IP, immunoprecipitation.

ubiquitination of MYB96 by MIEL1 in vivo, we carried out Co-IP analysis. Total protein extracts from 35S:MYB96-MYC and 35S:MYB96-MYCxmiel1-1 seeds were immunoprecipitated with an anti-MYC antibody. The precipitates were subsequently used for western blot analysis with anti-Ub or anti-MYC antibody. Co-IP analysis revealed that MYB96 was subjected to ubiquitination in wild-type background, but the ubiquitination of MYB96 was substantially reduced in miel1-1 background (Fig. 3a), supporting that MIEL1 stimulates ubiquitination of MYB96.

MYB96 ubiquitination may lead to its proteasomal degradation. To investigate this possibility, we employed MG132, a potent chemical inhibitor of the $26 \mathrm{~S}$ proteasome, and investigated its effects on MYB96 stability. Immunoblot analysis showed that MYB96 abundance was significantly elevated upon the treatment with MG132 in seeds (Fig. 3b). For comparison, we also measured MYB96 accumulation in seedlings after MG132 application and found that ubiquitination-dependent MYB96 degradation was also relevant in seedlings (Supplementary Fig. 7). Furthermore, we analysed MYB96 accumulation in seeds and seedlings of 35S:MYB96-MYC and 35S:MYB96-MYCxmiel1-1 genotypes. As expected, MYB96 stability was increased in miel1-1 background, regardless of plant developmental stages (Fig. 3c; Supplementary Fig. 8), even though transcript levels of MYB96 were similar in both genotypes (Supplementary Fig. 9).

It was reported that MYB96 regulates ABA-dependent seed germination by promoting $A B I 4$ transcription $^{42}$. We therefore postulated that MIEL1 might also influence $A B I 4$ expression in 

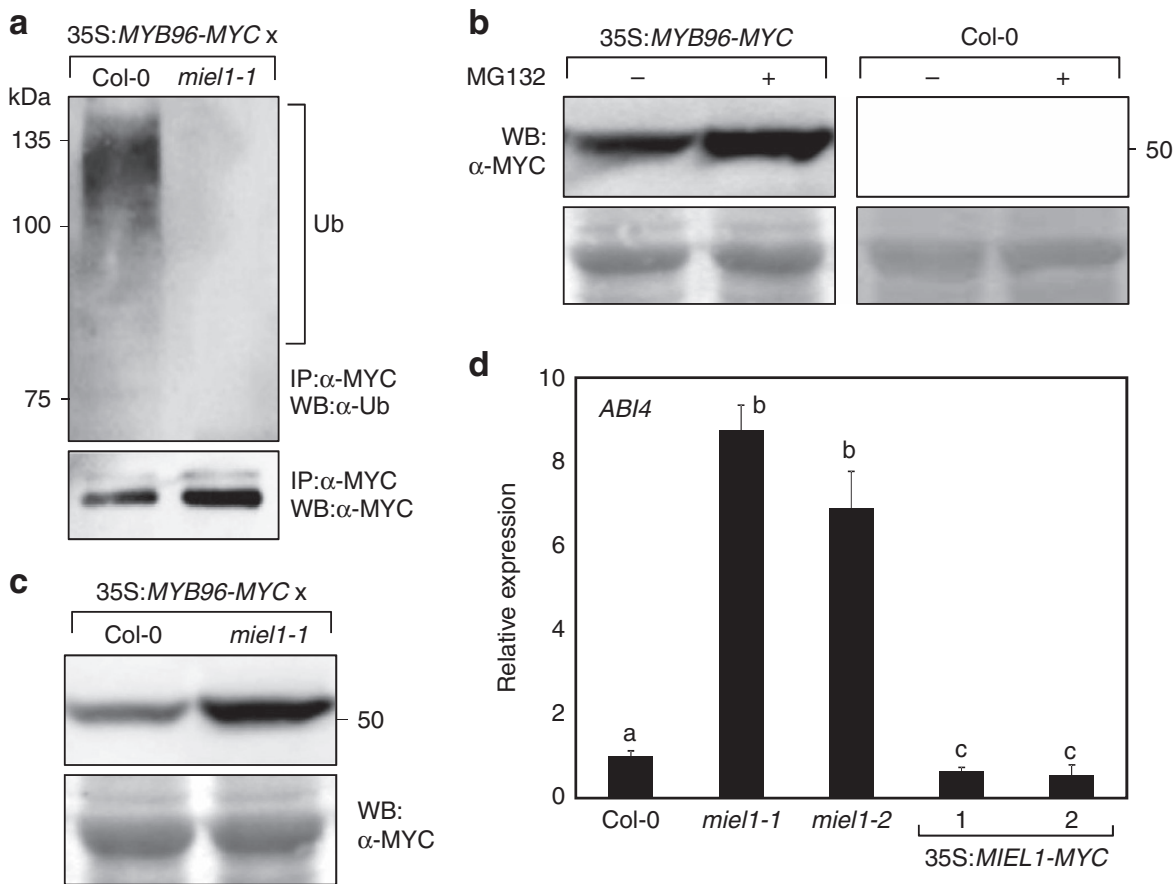

Figure 3 | Ubiquitination-induced proteolysis of MYB96 by MIEL1. (a) Ubiquitination of MYB96 by MIEL1 in seeds. Stratified seeds were pretreated with $50 \mu \mathrm{M}$ MG132 for $24 \mathrm{~h}$ before harvesting and used for Co-IP assays. Total protein extracts were immunoprecipitated using anti-MYC antibodies coupled to sepharose beads and were subjected to western blot analysis using anti-ubiquitin (top panel) and anti-MYC (bottom panel) antibodies. IP, immunoprecipitation; Ub, ubiquitinated MYB96 proteins; WB, western blot analysis. (b) Effects of MG132 on MYB96 stability. Stratified

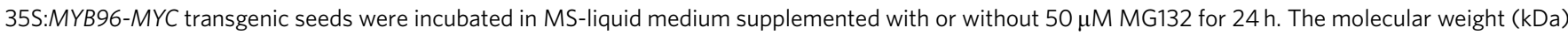
was indicated on the right side of the gel. (c) MYB96 accumulation in miel1-1. Stratified seeds were used for total protein extraction. MYB96 proteins were detected immunologically using an anti-MYC antibody. (d) ABI4 expression in seeds. Transcript accumulation was analysed by RT-qPCR. The elF4a gene was used as an internal control. Biological triplicates were averaged. Different letters represent a significant difference at $P<0.05$ (one-way analysis of variance with Fisher's post hoc test). Bars indicate the s.e.m.

seeds, possibly through protein degradation of MYB96. Consistent with the previous observations ${ }^{42}$, expression of $A B I 4$ was upregulated in miel1 mutant seeds, but downregulated in 35S:MIEL1-MYC transgenic seeds (Fig. 3d). Moreover, considering the MYB96 degradation by MIEL1 in seedlings, it was supposed that MIEL1 also affects expression of ABAand abiotic stress-responsive genes regulated by MYB96 during vegetative stages ${ }^{15-17,40}$. Indeed, HEPTAHELICAL TRANSMEMBRANE PROTEIN 1 (HHP1), 3-KETOACYL-COA SYNTHASE 6 (KCS6) and RD22 genes, which are directly controlled by MYB96 (refs 15,16,40), were significantly upregulated in miel1 mutant, but repressed in 35S:MIEL1-MYC transgenic seedlings (Supplementary Fig. 10), indicating that MIEL1 negatively regulates MYB96 stability by means of protein ubiquitination not only during seed germination, but also vegetative stages.

Ubiquitinated proteins are often subject to degradation by the $26 \mathrm{~S}$ proteasome. Of its two major particles, the $20 \mathrm{~S}$ core protease and the 19S regulatory particle (RP), the RP confers substrate specificity to the holoenzyme for their breakdown ${ }^{44}$. Notably, the REGULATORY PARTICLE NON-ATPASE 10 (RPN10) protein, a base component of the RP, plays a role in ABA signalling attenuation by promoting ubiquitination-derived protein turnover of ABA signalling components ${ }^{45,46}$. Thus, we asked whether the MIEL1 function is associated with RPN10. Quantitative PCR with reverse transcription (RT-qPCR) analysis showed that MYB96-regulated genes such as $A B I 4$, KCS6 and BETA-KETOACYL REDUCTASE 1 were unaffected, or rather suppressed, in rpn10-1 (Supplementary Fig. 11), suggesting that the MIEL1-MYB96 module is functionally independent of
RPN10 and might require different member(s) of the RP for the ubiquitinated MYB96 recognition. However, we cannot completely rule out the possible association of RPN10 with MIEL1 signalling in that transcript accumulation of MIEL1 was significantly increased in rpn10-1, as the ABI5 gene was altered (Supplementary Fig. 12), and RPN10 plays a fundamental role in ABA signalling ${ }^{45}$.

ABA suppresses MIEL1 but promotes MYB96 accumulation. The ABA sensitivity phenotype and altered accumulation of MYB96 in miel1 mutants suggest that a MIEL1-MYB96 signalling module may be required for proper ABA signal transduction. We therefore asked how ABA regulates accumulation patterns of MIEL1 and MYB96. To address this question, we first analysed the transcript accumulation of MIEL1. While expression of MIEL1 exhibited diurnal fluctuation under normal growth conditions (Supplementary Fig. 13) consistent with the light promotion of seed germination, exogenous ABA treatment significantly reduced its transcript accumulation both in seeds and seedlings (Fig. 1c; Supplementary Fig. 14). In accordance with this, protein accumulation of MIEL1 was also decreased in the presence of ABA (Fig. 4a; Supplementary Fig. 15), and its degradation was dependent on $26 \mathrm{~S}$ proteasomal activity (Fig. 4b; Supplementary Fig. 16). Accumulation of MYB96 protein was complementary to that of MIEL1. ABA promoted MYB96 accumulation, possibly due to the derepression from the destructive activity of MIEL1 (Fig. 4a; Supplementary Fig. 15).

In support of this, ubiquitination of MYB96 was significantly reduced upon ABA treatment (Fig. 4c; Supplementary Fig. 17). 

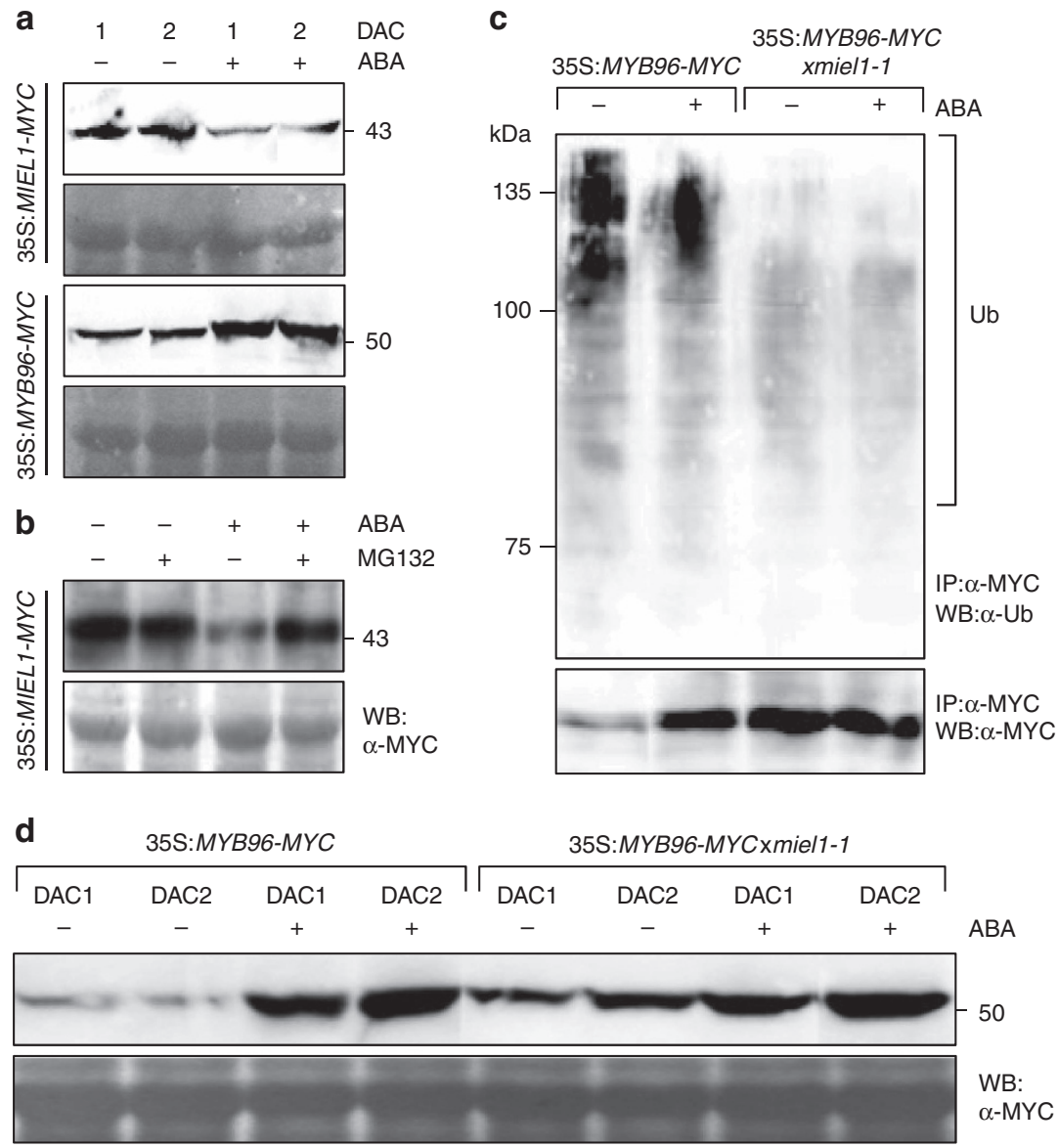

Figure 4 | ABA regulation of MIEL1 and MYB96 stability. (a) Protein abundance of MIEL1 and MYB96 in the presence of ABA. Seeds were germinated on MS medium supplemented with or without $1 \mu \mathrm{M} \mathrm{ABA}$ and incubated for the indicated time periods after cold stratification. The molecular weight ( $\mathrm{kDa}$ ) was indicated on the right side of the gel. (b) Effects of MG132 on ABA-dependent MIEL1 degradation. Stratified seeds were treated with $1 \mu M$ ABA and/or $50 \mu \mathrm{M} \mathrm{MG} 132$ for $24 \mathrm{~h}$. (c) Changes in ubiquitination of MYB96 in the presence of ABA. Stratified seeds were treated with $1 \mu \mathrm{M}$ ABA for $24 \mathrm{~h}$ and used for the total protein extraction. (d) Effects of ABA on MYB96 stability in miel7-1. Seeds were germinated on MS medium supplemented with or without $1 \mu \mathrm{M} \mathrm{ABA}$ and incubated for the indicated time periods after cold stratification. DAC, days after cold stratification; IP, immunoprecipitation; Ub, ubiquitinated MYB96 proteins; WB, Western blot analysis.

The reduction of its ubiquitination was correlated to the MIEL1 activity. Ubiquitination levels of MYB96 were markedly low and insensitive to ABA in miel1-1 background (Fig. 4c; Supplementary Fig. 17).

To further support the negative regulation of MYB96 by MIEL1 in the presence of ABA, we also examined the effects of ABA on MYB96 stability in miel1-1 background. As expected, exogenous treatment with ABA led to the increased accumulation of MYB96 in wild-type background (Fig. 4d; Supplementary Fig. 18), whereas a high level of MYB96 accumulation was observed even in the absence of $\mathrm{ABA}$ and the protein levels were less sensitive to ABA treatment in the miel1-1 mutant (Fig. $4 \mathrm{~d}$; Supplementary Fig. 18).

MYB96 is epistatic to MIEL1 in control of seed germination. To confirm the genetic hierarchy between MIEL1 and MYB96, we crossed the miell-1 mutant with myb96-1 and measured the germination percentage. In the presence of $\mathrm{ABA}$, germination of miel1-1 seeds was significantly delayed, whereas myb96-1 seeds exhibited reduced sensitivity to ABA compared with wild-type seeds (Fig. 5a). Notably, the germination percentage of miel1-1xmyb96-1 seeds was comparable to that of myb96-1 seeds (Fig. 5a). In agreement with this, transcript accumulation of $A B I 4$ in miel1-1xmyb96-1 was also similar to that of myb96-1 (Fig. 5b). Furthermore, no feedback regulation between MYB96 and MIEL1 was observed (Supplementary Fig. 19), providing further evidence that MYB96 is epistatic to MIEL1 and that they act in the same genetic pathway to control ABA-dependent seed germination.

MIEL1 may facilitate ABA and defence signalling crosstalk. MIEL1 largely depends on MYB96 in the control of seed germination (Fig. 5 and see Discussion). However, given that MIEL1 additionally regulates protein stability of a hypersensitive response (HR) regulator MYB30 in response to pathogen infection $^{34}$ and that MYB96 degradation by MIEL1 is also relevant in seedlings, MIEL1 might act in crosstalk between ABA and biotic stress signalling in vegetative tissues. To test this possibility, we examined the effects of ABA on MYB30-mediated signalling in seedlings. Notably, MYB30 turnover was suppressed by ABA in a MIEL1-dependent manner (Fig. 6a). Consistently, the ECERIFERUM 2 (CER2) and CER10 genes regulated by MYB30 during pathogen infection were considerably induced by ABA, and their expression was less sensitive to exogenous ABA treatment in the miel1-1 mutant (Fig. 6b).

In addition, the ABA signalling component MYB96 is also associated with the plant defence against biotrophs. Wild-type 

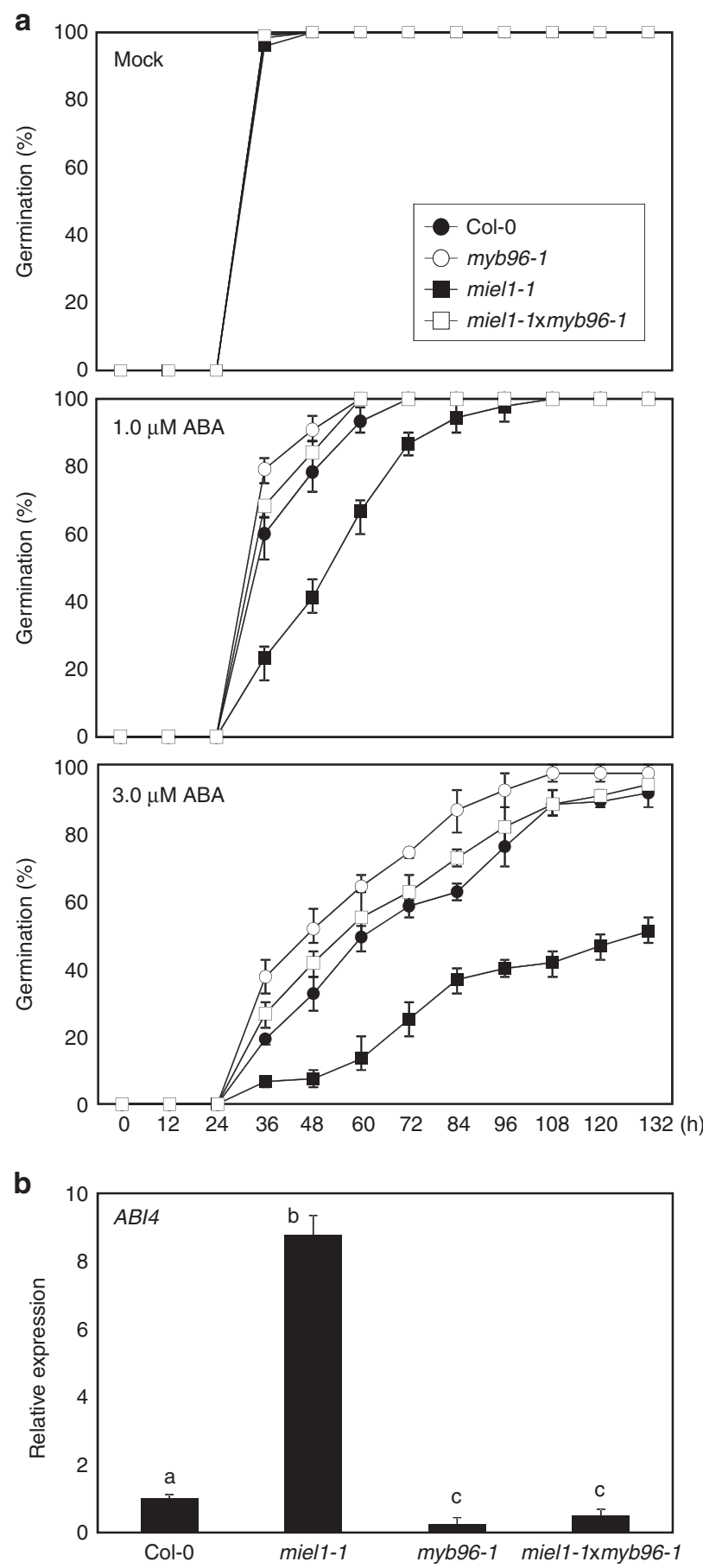

Figure 5 | Genetic hierarchy of MIEL1 and MYB96 in the control of seed germination. (a) Germination percentage of miel1-7xmyb96-1. Seed germination percentage of each genotype on different concentrations of $A B A$ was scored at the indicated time points after cold stratification. At least 50 seeds per genotype were measured in each replicate. Biological triplicates were averaged. Bars indicate the s.e.m. (b) $A B / 4$ expression in miel1-1xmyb96-1. Stratified seeds were used to analyse transcript accumulation. The elF4a gene was used as an internal control. Biological triplicates were averaged. Different letters represent a significant difference at $P<0.05$ (one-way analysis of variance with Fisher's post hoc test). Bars indicate the s.e.m.

plants infected with the avirulent Pseudomonas syringae pv. tomato DC3000/avrRpm1 (Pst DC3000/avrRpm1) cells showed increased protein accumulation of MYB96 (Fig. 6c), which may be due to the reduced levels of MIEL1 (ref. 34). MYB96 accumulation was not further responsive to pathogen infection in the miel1-1 mutant (Fig. 6c). Consistently, MYB96-regulated ABA-inducible genes HHP1, KCS6, RD22 and SALICYLIC ACID INDUCTION DEFICIENT 2 were induced by Pst DC3000/ avrRpm1 infection, and miel1 mutants showed constitutive high expression of them even in normal conditions (Fig. 6d).

Taken together, our findings indicate that MIEL1 is a novel ABA signalling mediator that promotes protein turnover of MYB96. This E3 Ub ligase has distinct molecular functions depending on the developmental stages. We propose that the MIEL1-MYB96 module regulates ABA signalling during seed germination, independently of MYB30 (see Discussion). In the seedling stages, we propose that MIEL1 stimulates protein turnover of both MYB96 and MYB30, which could potentially be involved in the coordination of plant responses to abiotic and biotic stresses (Fig. 6e).

\section{Discussion}

Eukaryotic E3 Ub ligases are classified into two major groups: single-subunit E3 ligases and multi-subunit E3 ligases. Singlesubunit E3 ligases include the Homologous to E6-AP Carboxyl Terminus, U-box and RING domain-containing proteins ${ }^{20,23}$. It is noteworthy that RING-finger proteins comprise the largest subgroup, and a significant number of RING proteins are involved in the control of ABA signalling (see above).

In the formation of multi-subunit E3 ligases, CULLIN (CUL) proteins act as a scaffold for assembling Ub E3 ligase complex machineries and recruit several components, including a RING-finger protein REGULATOR OF CULLINS1/RINGBOX1 and a substrate-recognition module composed of adaptor and substrate receptor proteins ${ }^{23,47}$. For example, CUL1 or CUL2 constitutes an S-PHASE KINASE-ASSOCIATED PROTEIN 1 (SKP1)-CUL-F-box-type complex along with the ArabidopsisSKP1-like adaptor protein and an F-box substrate receptor protein $^{47,48}$

Multi-subunit E3 ligases also participate in ABA signal transduction. The CUL1-interacting DROUGHT TOLERANCE REPRESSOR (DOR) F-box protein mediates ABA regulation of drought stress responses ${ }^{49}$. The DOR-deficient mutants show enhanced drought tolerance with hypersensitive stomatal closure, whereas ectopic expression of $D O R$ results in reduced drought tolerance.

CUL4 interacts with the DAMAGED DNA BINDING 1 (DDB1) adaptor protein and DDB1-BINDING WD40 PROTEIN (DWD)/DDB1-CUL4-ASSOCIATED FACTOR (DCAF) substrate receptor protein ${ }^{49,50}$. The Arabidopsis genome encodes $\sim 85$ DWD candidates and additional 34 WDxR motifcontaining proteins that do not contain an entire DWD box, but which are sufficient to interact with DDB1 (ref. 51). Notably, some of them are involved in ABA signal transduction. DWD HYPERSENSITIVE TO ABA1 (DWA1) and DWA2 regulate protein turnover of ABI5 (ref. 52). Consistently, dwa1dwa2 double mutants exhibit altered ABA sensitivity in root growth and seed germination ${ }^{52}$. DWA3 is also known to negatively regulate ABA signal transduction through an ABI5-independent pathway ${ }^{53}$. In addition, the ABA-inducible ABA-HYPERSENSITVE DCAF1 (ABD1) protein also plays a significant role in controlled proteolysis of ABI5. The abd1deficient mutants are hypersensitive to ABA in the control of seed germination, early seedling growth and drought tolerance ${ }^{54}$.

The CONSTITUTIVE PHOTOMORPHOGENIC10 (COP10)DEETIOLATED1 (DET1)-DDB1 complex (CDD complex), together with a part of the substrate adaptor module DET1-, DDB1-ASSOCIATED1 (DDA1) ${ }^{55}$, cooperates with the CUL4-E3 ligase in the control of ABA signalling ${ }^{56}$. DDA1 mediates 
a

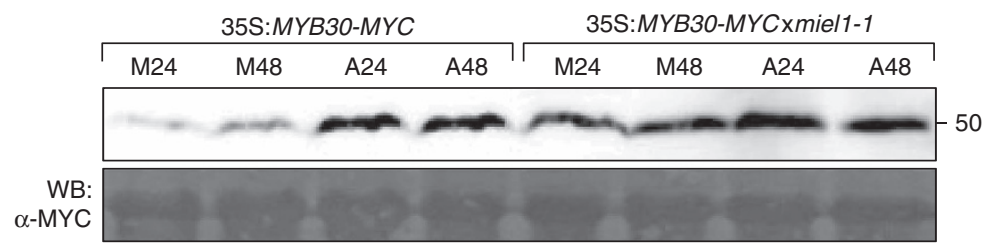

C

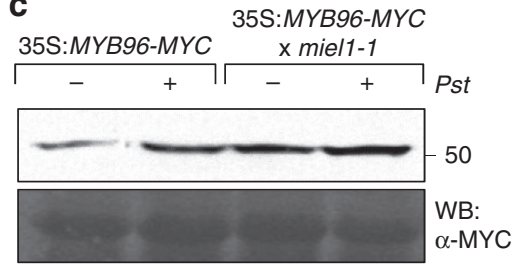

b
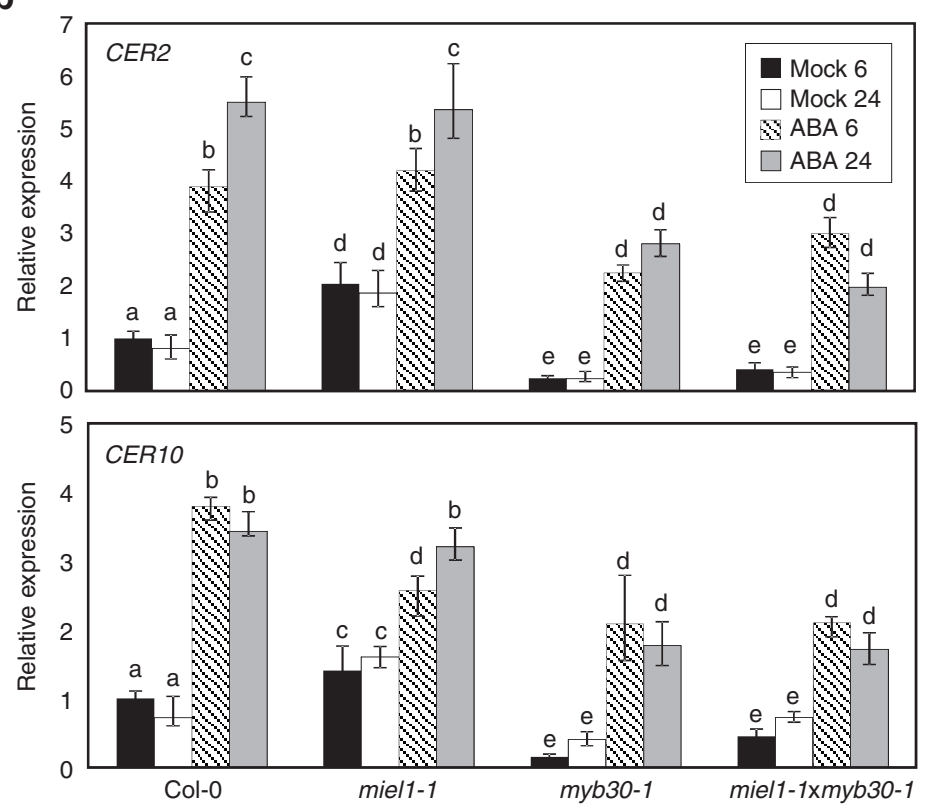

e

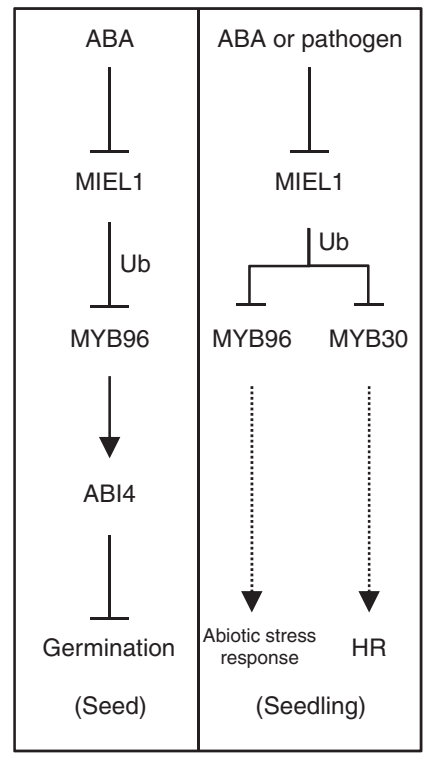

d

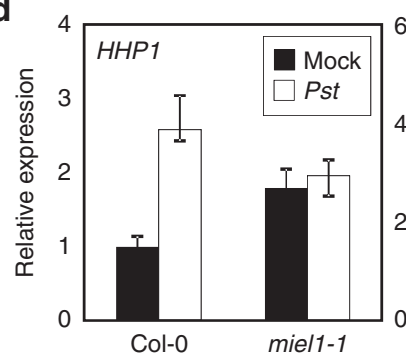

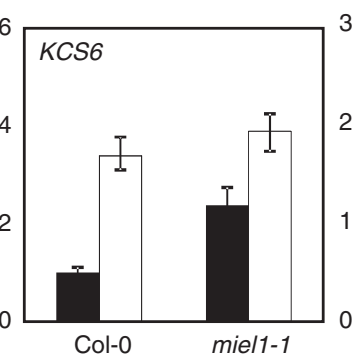

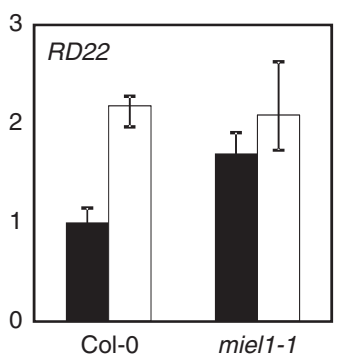

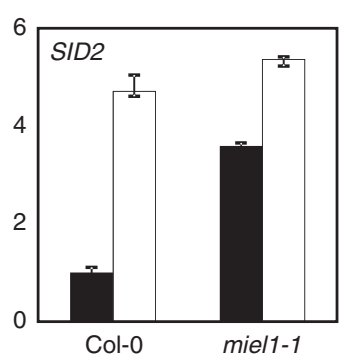

Figure 6 | Coordinated regulation of MYB96 and MYB30 by MIEL1 in seedlings. (a) Effects of ABA on MYB30 stability in mie/1-1. Two-week-old plants were incubated in MS-liquid medium supplemented with or without $20 \mu \mathrm{M} \mathrm{ABA}$ for the indicated time periods (h). MYB30 proteins were detected immunologically using an anti-MYC antibody. The molecular weight $(\mathrm{kDa})$ was indicated on the right side of the gel. (b) Effects of $A B A$ on transcript accumulation of MYB30 target genes. Two-week-old plants were incubated in MS-liquid medium supplemented with or without $20 \mu \mathrm{M}$ ABA for the indicated time periods (h). Transcript accumulation was analysed by RT-qPCR. The elF4a gene was used as an internal control. Biological triplicates were averaged. Different letters represent a significant difference at $P<0.05$ (one-way analysis of variance with Fisher's post hoc test). Bars indicate the s.e.m. (c) Effects of pathogen infection on MYB96 stability in miel1-1. Four-week-old plants were infected with Pst DC3000/avrRpm1 (Pst), and the infected leaves were used for total protein isolation. (d) Effects of Pst infection on transcript accumulation of MYB96 target genes. Four-week-old plants were infected with Pst, and the infected leaves were used to analyse transcript accumulation. Biological triplicates were averaged. Bars indicate the s.e.m. (e) Proposed roles of MIEL1 in Arabidopsis. In seeds, MIEL1 stimulates protein turnover of MYB96, a positive regulator of ABA signalling, to promote seed germination. In the presence of ABA, MIEL1 stability is reduced, and thus MYB96 is derepressed to activate ABA signaling cascades. In seedling stages, MIEL1 may facilitate a crosstalk between abiotic and biotic signaling by regulating protein turnover of both MYB96 and MYB30, which mediate ABA-dependent drought tolerance and hypersensitive defense responses (HR), respectively. The cross-regulation probably facilitates to efficiently trigger both abiotic and biotic responses under environmentally unfavourable conditions. Ub, ubiquitination.

proteolytic degradation of the PYL8 ABA receptor, and DDA1 activity is attenuated in the presence of $\mathrm{ABA}^{56}$. Consistently, a variety of developmental processes mediated by ABA, including seed germination, early seedling growth and root growth, are altered in plants mis-expressing DDA1 or CDD complex components ${ }^{56}$. Collectively, controlled proteolysis is a key molecular scheme in ABA signalling, and specific combinations of E3 ligases and $\mathrm{ABA}$ signalling components facilitate precise signal transduction under environmental stress conditions.

MYB96 is a representative ABA signalling mediator that regulates a variety of physiological processes, such as shoot and root development, seed germination and dormancy, cuticular wax biosynthesis, hormone homoeostasis and stress tolerance, under environmentally unfavourable conditions ${ }^{15-17,40-42}$. Considering 
its myriad of regulatory targets, MYB96 may act on upstream effector of the ABA signalling pathway.

Despite its importance in $\mathrm{ABA}$ signalling, the upstream regulators of MYB96 are largely unknown. Here we found that MIEL1 is an regulator of MYB96, which stimulates its proteolytic degradation. MIEL1 can interact with MYB96 and promote its ubiquitination. We propose that in the absence of ABA, MIEL1 accumulates and targets MYB96 for degradation to attenuate $\mathrm{ABA}$ signal transduction, whereas in the presence of $\mathrm{ABA}$, protein accumulation of MYB96 is elevated, because MIEL1 is degraded by $26 \mathrm{~S}$ proteasome activity. Consistent with an inhibitory role of MIEL1 in ABA signalling, 35S:MIEL1-MYC transgenic seeds were hyposensitive to ABA, while miell-deficient mutant seeds exhibited increased sensitivity to ABA during the germination process.

The MIEL1-MYB96 module is important for regulating ABA-dependent seed germination. While MYB30, another regulatory target of MIEL1, also plays a role in seed germination, its function is most likely independent of MIEL1. Compared with the promotive role of MIEL1 in seed germination, MYB30 also stimulates seed germination in the presence of $\mathrm{ABA}^{57}$. The MYB30-deficient myb30-1 mutant seeds are hypersensitive, whereas MYB30-overexpressing transgenic seeds exhibit reduced sensitivity to $A B A^{57}$, which do not suggest a role for MYB30 degradation by MIEL1 during seed germination. In support of this, genetic analysis revealed that MYB96 is epistatic to MIEL1, with respect to the regulation of ABA-dependent seed germination (Fig. 5).

The MIEL1 gene is also regulated by light, in addition to ABA. Furthermore, it is strongly and specifically expressed in micropylar endosperm, which plays a decisive role in endosperm weakening and thus seed germination ${ }^{37,38}$. These observations raise the possibility that MIEL1 might be a crucial molecular component of seed germination process and that MYB96 may not be a sole target of MIEL1 in seeds. For instance, the ELONGATED HYPOCOTYL 5 (HY5) bZIP transcription factor is a plausible candidate, which mediates not only light signalling but also ABA response ${ }^{58}$. HY5 is responsible for the activation of many light-regulated genes and also regulates $\mathrm{ABA}$ sensitivity by activating ABI5 and ABI5-regulated LATE EMBRYOGENESIS$A B U N D A N T$ genes in seeds ${ }^{58}$. Consistently, the hy5 mutants exhibit reduced sensitivity to ABA during seed germination ${ }^{58,59}$, and $\mathrm{ABA}$ enhances light responses and inhibits hypocotyl elongation in early seedlings ${ }^{58,59}$. Although further works are required, MIEL1 might act as an integrator of light and $A B A$ signalling to coordinate proper timing to germinate.

Molecular actions of MIEL1 somewhat differ depending on the plant developmental stages. While MIEL1 primarily acts via MYB96 in the control of seed germination, our data suggest that it may act in crosstalk of $\mathrm{ABA}$ and defence signalling during the vegetative stages by promoting protein turnover of both MYB96 and MYB30. On one hand, MIEL1 attenuates defence responses by promoting MYB30 degradation under normal growth condition, but its expression is suppressed upon the exposure to pathogen attack to allow MYB30 accumulation and hypersensitive responses ${ }^{34}$. Notably, the MYB30-mediated defence responses were also stimulated by $\mathrm{ABA}$, presumably due to ABA-dependent MIEL1 degradation. On the other hand, MIEL1 also shapes the MYB96 activity in parallel with the MYB30 pathway. Accumulation of MYB96 levels was enhanced by both ABA treatment and pathogen infection (Fig. 6c). Consistently, we found that MYB96 target genes that are known to be regulated by $\mathrm{ABA}^{15,16,40}$ were also activated in response to pathogen infection.

On the basis of these results, we suggest that MIEL1 may possibly coordinate $\mathrm{ABA}$ and defence signalling. It has been proposed that ABA-mediated stress responses are closely associated with defence responses ${ }^{60}$. Abiotic stress can trigger defence responses to prime upcoming pathogen attacks ${ }^{61}$, and pathogen invasion also induces proper defence responses, as well as abiotic stress responses to ensure plant fitness and adaptation to multiple ambient stresses. MIEL1 may play a role in this crosstalk, contributing to the response to both $\mathrm{ABA}$ and biotic stresses to establish the efficient protective responses.

\section{Methods}

Plant materials and growth conditions. Arabidopsis thaliana (Columbia-0 ecotype) was used for all experiments unless otherwise specified. Plants were grown under long-day conditions (LDs; 16-h light/8-h dark cycles) with cool white fluorescent light $\left(100 \mu \mathrm{mol}\right.$ photons $\left.\mathrm{m}^{-2} \mathrm{~s}^{-1}\right)$ at $23^{\circ} \mathrm{C}$. The myb96-ox and myb96-1 mutants (GABI_120B05) were previously reported ${ }^{15-17}$. The miel1-1 (SALK_097638) and miel1-2 (SALK_041369) mutants were isolated from a T-DNA insertional mutant pool deposited in the Arabidopsis Biological Resource Center (https://abrc.osu.edu/).

To produce transgenic plants overexpressing the MIEL1 gene, a full-length cDNA was amplified with the primer pair (F: 5'-GAGGCGCGCCATGGAAGCT TCACCCAATG /R: 5'-GAGACGTCTGTTGAGGAAGAACAGGAGGC) and then subcloned into the binary pBA002 vector under the control of the cauliflower mosaic virus $35 \mathrm{~S}$ promoter. Agrobacterium tumefaciens-mediated Arabidopsis transformation was then performed.

Seed germination assays. All genotypes were grown at $23^{\circ} \mathrm{C}$ under LDs, and seeds were collected at the same time. Harvested seeds were dried at room temperature at least 1 month before germination assays. For seed germination assays, 40-50 seeds for each line were sterilized and plated on MS medium (half-strength MS salts, $0.05 \%$ MES, pH 5.7 and $1 \%$ agar) supplemented with various

concentrations of $\mathrm{ABA}(0,1,3 \mu \mathrm{M})$. Plates were stratified in darkness for 3 days at $4{ }^{\circ} \mathrm{C}$ and transferred to a culture room set at $23^{\circ} \mathrm{C}$ with a 16 -h light/8-h dark cycle. Germination was scored at the indicated time points by counting the frequency of radicle emergence from the seed coat and endosperm. For each germination assay, biological triplicates were performed.

Quantitative real-time RT-PCR analysis. Total RNA was extracted using TRI reagent (TAKARA Bio, Singa, Japan) according to the manufacturer's recommendations. RT was performed using Moloney Murine Leukaemia Virus reverse transcriptase (Dr Protein, Seoul, South Korea) with oligo (dT18) to synthesize first-strand cDNA from $2 \mu \mathrm{g}$ of total RNA. Total RNA samples were pretreated with an RNAse-free DNAse. cDNAs were diluted to $100 \mu \mathrm{l}$ with TE buffer, and $1 \mu \mathrm{l}$ of diluted cDNA was used for PCR amplification.

Quantitative RT-PCR reactions were performed in 96-well blocks using the Step-One Plus Real-Time PCR System (Applied Biosystems). The PCR primers used are listed in Supplementary Table 1. The values for each set of primers were normalized relative to the EUKARYOTIC TRANSLATION INITIATION FACTOR $4 A 1$ (eIF4A) gene (At3g13920). All RT-qPCR reactions were performed with biological triplicates using total RNA samples extracted from the three independent replicate samples. The comparative $\Delta \Delta C_{\mathrm{T}}$ method was employed to evaluate relative quantities of each amplified product in the samples. The threshold cycle $\left(C_{\mathrm{T}}\right)$ was automatically determined for each reaction with the analysis software set using default parameters. The specificity of the RT-qPCR reactions was determined by melt curve analysis of the amplified products using the standard method employed by the software.

Y2H assays. $\mathrm{Y} 2 \mathrm{H}$ assays were performed using the BD Matchmaker system (Clontech, Mountain View, CA, USA). The pGADT7 vector was used for the GAL4 $\mathrm{AD}$ fusion, and the pGBKT7 vector was used for GAL4 BD fusion. The yeast strain AH109 harbouring the $L a c Z$ and His reporter genes was used. PCR products were subcloned into the pGBKT7 and pGADT7 vectors. The expression constructs were co-transformed into yeast AH109 cells and transformed cells were selected by growth on SD/-Leu/-Trp medium and SD/-Leu/-Trp/-His/-Ade. Interactions between proteins were analysed by measuring the $\beta$-galactosidase activity using $o$-nitrophenyl- $\beta$-D-galactopyranoside as a substrate.

BiFC assays. BiFC assays were performed as described previously ${ }^{62}$. In brief, the MYB96 gene was fused in-frame to the $5^{\prime}$ end of a gene sequence encoding the C-terminal half of EYFP in the pSATN-cEYFP-C1 vector (E3082). The MIEL1 cDNA sequence was fused in-frame to the $5^{\prime}$ end of a gene sequence encoding the N-terminal half of EYFP in the pSATN-nEYFP-C1 vector (E3081). Expression constructs were co-transformed into Arabidopsis protoplasts. Expression of the fusion constructs was monitored by fluorescence microscopy using a Zeiss LSM510 confocal microscope (Carl Zeiss, Jena, Germany).

Co-IP assays. A. tumefaciens cells containing MIEL1-MYC and MYB96-GFP constructs were injected to 3 -week-old $N$. benthamiana leaves. Tobacco leaves were 
homogenized in protein extraction buffer $(25 \mathrm{mM}$ Tris- $\mathrm{HCl}, \mathrm{pH} 7.5,150 \mathrm{mM} \mathrm{NaCl}$, $5 \%$ glycerol, $0.05 \%$ Nonidet P-40, $2.5 \mathrm{mM}$ EDTA, $1 \mathrm{mM}$ phenylmethylsulfonyl fluoride and $1 \times$ complete cocktail of protease inhibitors). After protein extraction, anti-MYC antibodies (05-724, Millipore, Billerica, MA, USA) coupled to Protein-A sepharose beads (Sigma-Aldrich, St Louis, MO, USA) were mixed and incubated for $4 \mathrm{~h}$ at $4{ }^{\circ} \mathrm{C}$. The precipitated samples were washed at least four times with the protein extraction buffer and then eluted by $1 \times$ SDS-polyacrylamide gel electrophoresis (PAGE) loading buffer to subject to SDS-PAGE with anti-MYC (1:2000 dilution; Millipore) or anti-GFP antibodies (1:1,000 dilution; sc-9996, Santa Cruz Biotech., Dallas, Texas, USA). The original gel images are shown in Supplementary Fig. 20.

Treatment of seedlings with ABA. For treatment with ABA, 2-week-old seedlings grown under LDs were transferred to half-strength MS-liquid medium supplemented with $20 \mu \mathrm{M}(+)$-cis,trans-ABA (L06278; Alfa Aesar, Ward Hill, MA, USA).

Immunoblot analysis. Harvested plant materials were ground in liquid nitrogen and total cellular extracts were suspended in SDS-PAGE sample loading buffer. The protein samples were then analysed by SDS/PAGE (10\% gels) and blotted onto polyvinylidene difluoride membranes (Roche, Indianapolis, IN, USA). Epitope-tagged proteins were immunologically detected using anti-MYC (1:2,000 dilution; Millipore) or anti-Ub antibodies (1:1,000 dilution; sc-0817, Santa Cruz Biotech., Dallas, Texas, USA). Bands from at least three independent blots were quantified using Image J software (http://imagej.nih.gov) and averaged. The original gel images are shown in Supplementary Fig. 20

Ubiquitination assays. Plant materials were pretreated with $50 \mu \mathrm{M}$ MG132 (Calbiochem, Darmstadt, Germany) for $24 \mathrm{~h}$ and used for nuclear extraction. Nuclear extracts and anti-MYC antibodies coupled to Protein-A sepharose beads (Sigma-Aldrich) were mixed in extraction buffer $(20 \mathrm{mM}$ Tris, pH 7.4, $100 \mathrm{mM}$ sodium chloride, $0.5 \%$ Nonidet P-40, $0.5 \mathrm{mM}$ EDTA, $0.5 \mathrm{mM}$ PMSF and protease inhibitor cocktail) containing $50 \mu \mathrm{M}$ MG132 and incubated for $2 \mathrm{~h}$ at $23^{\circ} \mathrm{C}$. The beads were recovered by centrifugation and washed with extraction buffer. The bound proteins were eluted with $1 \times$ SDS-PAGE loading buffer and subjected to SDS-PAGE. Immunological analysis was performed using anti-Ub (1:1,000 dilution; Santa Cruz Biotech.) and anti-MYC antibodies (1:2,000 dilution; Millipore)

Pathogen infection. Four-week-old plants grown in soil under LDs were used for pathogen infection. The avirulent $P$. syringae pv. tomato DC3000/avrRpm 1 (Pst DC3000/avrRpm1) strain were cultured for $24 \mathrm{~h}$ at $27^{\circ} \mathrm{C}$ in Luria-Bertani medium supplemented with rifampicin $\left(50 \mathrm{mgl}^{-1}\right)$. A bacterial cell suspension was prepared at $\mathrm{OD}_{600}=0.02$ in $10 \mathrm{mM} \mathrm{MgCl} 2$ and pressure-infiltrated into the fourth and fifth rosette leaves. The inoculated plants were transferred to a growth chamber set at $23^{\circ} \mathrm{C}$ with relative humidity of $80 \%$ and further grown for 2 days under LDs.

Data availability. The authors declare that all data supporting the findings of this study are available in the manuscript and its supplementary files or are available from the corresponding author upon reasonable request.

\section{References}

1. Nakashima, K., Ito, Y. \& Yamaguchi-Shinozaki, K. Transcriptional regulatory networks in response to abiotic stresses in Arabidopsis and grasses. Plant Physiol. 149, 88-95 (2009).

2. Lopez-Molina, L., Mongrand, S. \& Chua, N. H. A postgermination developmental arrest checkpoint is mediated by abscisic acid and requires the ABI5 transcription factor in Arabidopsis. Proc. Natl Acad. Sci. USA 98, 4782-4787 (2001).

3. Finkelstein, R. R., Gampala, S. S. \& Rock, C. D. Abscisic acid signaling in seeds and seedlings. Plant Cell 14, S15-S45 (2002).

4. Nambara, E. \& Marion-Poll, A. Abscisic acid biosynthesis and catabolism. Annu. Rev. Plant Biol. 56, 165-185 (2005).

5. Yamaguchi-Shinozaki, K. \& Shinozaki, K. Transcriptional regulatory networks in cellular responses and tolerance to dehydration and cold stresses. Annu. Rev. Plant Biol. 57, 781-803 (2006).

6. Nishimura, N. et al. Structural mechanism of abscisic acid binding and signaling by dimeric PYR1. Science 326, 1373-1379 (2009).

7. Fujii, H. et al. In vitro reconstitution of an abscisic acid signalling pathway. Nature 462, 660-664 (2009).

8. Umezawa, T. et al. Molecular basis of the core regulatory network in ABA responses: sensing, signaling and transport. Plant Cell Physiol. 51, 1821-1839 (2010).

9. Qin, F., Shinozaki, K. \& Yamaguchi-Shinozaki, K. Achievements and challenges in understanding plant abiotic stress responses and tolerance. Plant Cell Physiol. 52, 1569-1582 (2011).
10. Shinozaki, K. \& Yamaguchi-Shinozaki, K. Molecular responses to dehydration and low temperature: differences and cross-talk between two stress signaling pathways. Curr. Opin. Plant Biol. 3, 217-223 (2000).

11. Abe, H. et al. Arabidopsis AtMYC2 (bHLH) and AtMYB2 (MYB) function as transcriptional activators in abscisic acid signaling. Plant Cell 15, 63-78 (2003).

12. Cominelli, E., Sala, T., Calvi, D., Gusmaroli, G. \& Tonelli, C. Over-expression of the Arabidopsis AtMYB41 gene alters cell expansion and leaf surface permeability. Plant J. 53, 53-64 (2008).

13. Jung, C. et al. Overexpression of AtMYB44 enhances stomatal closure to confer abiotic stress tolerance in transgenic Arabidopsis. Plant Physiol. 146, 623-635 (2008).

14. Ding, Z. et al. Transgenic expression of MYB15 confers enhanced sensitivity to abscisic acid and improved drought tolerance in Arabidopsis thaliana. J. Genet. Genomics 36, 17-29 (2009).

15. Seo, P. J. et al. The MYB96 transcription factor mediates abscisic acid signaling during drought stress response in Arabidopsis. Plant Physiol. 151, 275-289 (2009).

16. Seo, P. J. et al. The MYB96 transcription factor regulates cuticular wax biosynthesis under drought conditions in Arabidopsis. Plant Cell 23, 1138-1152 (2011).

17. Seo, P. J. \& Park, C. M. MYB96-mediated abscisic acid signals induce pathogen resistance response by promoting salicylic acid biosynthesis in Arabidopsis. New Phytol. 186, 471-483 (2010).

18. Cui, M. H. et al. An Arabidopsis R2R3-MYB transcription factor, AtMYB20, negatively regulates type $2 \mathrm{C}$ serine/threonine protein phosphatases to enhance salt tolerance. FEBS Lett. 587, 1773-1778 (2013).

19. Mazzucotelli, E. et al. The e3 ubiquitin ligase gene family in plants: regulation by degradation. Curr. Genomics 7, 509-522 (2006).

20. Lee, J. H. \& Kim, W. T. Regulation of abiotic stress signal transduction by E3 ubiquitin ligases in Arabidopsis. Mol. Cells 31, 201-208 (2011).

21. Sadanandom, A., Bailey, M., Ewan, R., Lee, J. \& Nelis, S. The ubiquitin-proteasome system: central modifier of plant signalling. New Phytol. 196, 13-28 (2012).

22. Smalle, J. \& Vierstra, R. D. The ubiquitin $26 \mathrm{~S}$ proteasome proteolytic pathway. Annu. Rev. Plant Biol. 55, 555-590 (2004).

23. Stone, S. L. et al. Functional analysis of the RING-type ubiquitin ligase family of Arabidopsis. Plant Physiol. 137, 13-30 (2005).

24. Callis, J. \& Vierstra, R. D. Protein degradation in signaling. Curr. Opin. Plant Biol. 3, 381-386 (2000).

25. Dreher, K. \& Callis, J. Ubiquitin, hormones and biotic stress in plants. Ann. Bot. 99, 787-822 (2007)

26. Bueso, E. et al. A forward genetic approach in Arabidopsis thaliana identifies a RING-type ubiquitin ligase as a novel determinant of seed longevity. Plant Sci. 215-216, 110-116 (2014)

27. Zhang, X., Garreton, V. \& Chua, N. H. The AIP2 E3 ligase acts as a novel negative regulator of $\mathrm{ABA}$ signaling by promoting ABI3 degradation. Genes Dev. 19, 1532-1543 (2005).

28. Stone, S. L., Williams, L. A., Farmer, L. M., Vierstra, R. D. \& Callis, J. KEEP ON GOING, a RING E3 ligase essential for Arabidopsis growth and development, is involved in abscisic acid signaling. Plant Cell 18, 3415-3428 (2006).

29. Liu, H. \& Stone, S. L. Abscisic acid increases Arabidopsis ABI5 transcription factor levels by promoting KEG E3 ligase self-ubiquitination and proteasomal degradation. Plant Cell 22, 2630-2641 (2010).

30. Qin, F. et al. Arabidopsis DREB2A-interacting proteins function as RING E3 ligases and negatively regulate plant drought stress-responsive gene expression. Plant Cell 20, 1693-1707 (2008).

31. Zhang, Y. et al. SDIR1 is a RING finger E3 ligase that positively regulates stress-responsive abscisic acid signaling in Arabidopsis. Plant Cell 19, 1912-1929 (2007).

32. Bu, Q. et al. The Arabidopsis RING finger E3 ligase RHA2a is a novel positive regulator of abscisic acid signaling during seed germination and early seedling development. Plant Physiol. 150, 463-481 (2009).

33. Li, H. et al. The Arabidopsis RING finger E3 ligase RHA2b acts additively with RHA2a in regulating abscisic acid signaling and drought response. Plant Physiol. 156, 550-563 (2011).

34. Marino, D. et al. Arabidopsis ubiquitin ligase MIEL1 mediates degradation of the transcription factor MYB30 weakening plant defence. Nat. Commun. $\mathbf{4}$, 1476 (2013).

35. Finch-Savage, W. E. \& Leubner-Metzger, G. Seed dormancy and the control of germination. New Phytol. 171, 501-523 (2006).

36. Dekkers, B. J. et al. Transcriptional dynamics of two seed compartments with opposing roles in Arabidopsis seed germination. Plant Physiol. 163, 205-215 (2013).

37. Müller, K., Tintelnot, S. \& Leubner-Metzger, G. Endosperm-limited Brassicaceae seed germination: abscisic acid inhibits embryo-induced endosperm weakening of Lepidium sativum (cress) and endosperm rupture of cress and Arabidopsis thaliana. Plant Cell Physiol. 47, 864-877 (2006). 
38. Linkies, A. et al. Ethylene interacts with abscisic acid to regulate endosperm rupture during germination: a comparative approach using Lepidium sativum and Arabidopsis thaliana. Plant Cell 21, 3803-3822 (2009).

39. Morris, K. et al. Regulation of seed germination in the close Arabidopsis relative Lepidium sativum: a global tissue-specific transcript analysis. Plant Physiol. 155, 1851-1870 (2011).

40. Lee, H. G. \& Seo, P. J. The MYB96-HHP module integrates cold and abscisic acid signaling to activate the CBF-COR pathway in Arabidopsis. Plant J. 82, 962-977 (2015)

41. Lee, H. G., Lee, K. \& Seo, P. J. The Arabidopsis MYB96 transcription factor plays a role in seed dormancy. Plant Mol. Biol. 87, 371-381 (2015).

42. Lee, K., Lee, H. G., Yoon, S., Kim, H. U. \& Seo, P. J. The Arabidopsis MYB96 transcription factor is a positive regulator of $A B I 4$ in the control of seed germination. Plant Physiol. 168, 677-689 (2015).

43. Liu, H. \& Stone, S. L. E3 ubiquitin ligases and abscisic acid signaling. Plant Signal. Behav. 6, 344-348 (2011).

44. Rosenzweig, R., Osmulski, P. A., Gaczynska, M. \& Glickman, M. H. The central unit within the 19 S regulatory particle of the proteasome. Nat. Struct. Mol. Biol. 15, 573-580 (2008).

45. Smalle, J. et al. The pleiotropic role of the $26 \mathrm{~S}$ proteasome subunit RPN10 in Arabidopsis growth and development supports a substrate-specific function in abscisic acid signaling. Plant Cell 15, 965-980 (2003).

46. Marshall, R. S., Li, F., Gemperline, D. C., Book, A. J. \& Vierstra, R. D. Autophagic degradation of the $26 \mathrm{~S}$ proteasome is mediated by the dual ATG8/ubiquitin receptor RPN10 in Arabidopsis. Mol. Cell 58, 1053-1066 (2015).

47. Thomann, A., Dieterle, M. \& Genschik, P. Plant CULLIN-based E3s: phytohormones come first. FEBS Lett. 579, 3239-3245 (2005).

48. Moon, J., Parry, G. \& Estelle, M. The ubiquitin-proteasome pathway and plant development. Plant Cell 16, 3181-3195 (2004).

49. Zhang, Y. et al. Arabidopsis DDB1-CUL4 ASSOCIATED FACTOR1 forms a nuclear E3 ubiquitin ligase with DDB1 and CUL4 that is involved in multiple plant developmental processes. Plant Cell 20, 1437-1455 (2008).

50. Lee, J. \& Zhou, P. DCAFs, the missing link of the CUL4-DDB1 ubiquitin ligase. Mol. Cell 26, 775-780 (2007).

51. Lee, J. H. et al. Characterization of Arabidopsis and rice DWD proteins and their roles as substrate receptors for CUL4-RING E3 ubiquitin ligases. Plant Cell 20, 152-167 (2008).

52. Lee, J. H. et al. DWA1 and DWA2, two Arabidopsis DWD protein components of CUL4-based E3 ligases, act together as negative regulators in ABA signal transduction. Plant Cell 22, 1716-1732 (2010).

53. Lee, J. H., Terzaghi, W. \& Deng, X. W. DWA3, an Arabidopsis DWD protein, acts as a negative regulator in ABA signal transduction. Plant Sci. 180, 352-357 (2011).

54. Seo, K. I. et al. ABD1 is an Arabidopsis DCAF substrate receptor for CUL4-DDB1-based E3 ligases that acts as a negative regulator of abscisic acid signaling. Plant Cell 26, 695-711 (2014).

55. Olma, M. H. et al. An interaction network of the mammalian COP9 signalosome identifies Dda1 as a core subunit of multiple Cul4-based E3 ligases. J. Cell Sci. 122, 1035-1044 (2009).

56. Irigoyen, M. L. et al. Targeted degradation of abscisic acid receptors is mediated by the ubiquitin ligase substrate adaptor DDA1 in Arabidopsis. Plant Cell 26, 712-728 (2014).

57. Zheng, Y., Schumaker, K. S. \& Guo, Y. Sumoylation of transcription factor MYB30 by the small ubiquitin-like modifier E3 ligase SIZ1 mediates abscisic acid response in Arabidopsis thaliana. Proc. Natl Acad. Sci. USA 109, 12822-12827 (2012).

58. Chen, H. et al. Integration of light and abscisic acid signaling during seed germination and early seedling development. Proc. Natl Acad. Sci. USA 105, 4495-4500 (2008).

59. Srivastava, A. K. et al. SHORT HYPOCOTYL IN WHITE LIGHT1 interacts with ELONGATED HYPOCOTYL 5 (HY5) and CONSTITUTIVE PHOTOMORPHOGENIC 1 (COP1) and promotes COP1-mediated degradation of HY5 during Arabidopsis seedling development. Plant Physiol. 169, 2922-2934 (2015).

60. Asselbergh, B., De-Vleesschauwer, D. \& Höfte, M. Global switches and fine-tuning-ABA modulates plant pathogen defence. Mol. Plant Microbe Interact. 21, 709-719 (2008).

61. Conrath, U., Pieterse, C. M. \& Mauch-Mani, B. Priming in plant-pathogen interactions. Trends Plant Sci. 7, 210-216 (2002).

62. Seo, P. J. et al. A self-regulatory circuit of CIRCADIAN CLOCK-ASSOCIATED1 underlies the circadian clock regulation of temperature responses in Arabidopsis. Plant Cell 24, 2427-2442 (2012).

\section{Acknowledgements}

We thank Dr Jan Smalle at University of Kentucky (Lexington, Kentucky, USA) for kindly providing rpn10-1 seeds. This work was supported by the Global Research Network (NRF-2014S1A2A2028392) program provided by the National Research Foundation of Korea and by the Next-Generation BioGreen 21 Program (PJ01109001) provided by the Rural Development Administration.

\section{Author contributions}

P.J.S. conceived and designed the experiments. P.J.S wrote the paper with the help of H.G.L. H.G.L. conducted experiments and contributed to the study design.

\section{Additional information}

Supplementary Information accompanies this paper at http://www.nature.com/ naturecommunications

Competing financial interests: The authors declare no competing financial interests.

Reprints and permission information is available online at http://npg.nature.com/ reprintsandpermissions/

How to cite this article: Lee, H. G. \& Seo, P. J. The Arabidopsis MIEL1 E3 ligase negatively regulates ABA signalling by promoting protein turnover of MYB96. Nat. Commun. 7:12525 doi: 10.1038/ncomms12525 (2016).

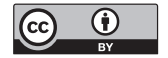

This work is licensed under a Creative Commons Attribution 4.0 International License. The images or other third party material in this article are included in the article's Creative Commons license, unless indicated otherwise in the credit line; if the material is not included under the Creative Commons license, users will need to obtain permission from the license holder to reproduce the material. To view a copy of this license, visit http://creativecommons.org/licenses/by/4.0/

(C) The Author(s) 2016 